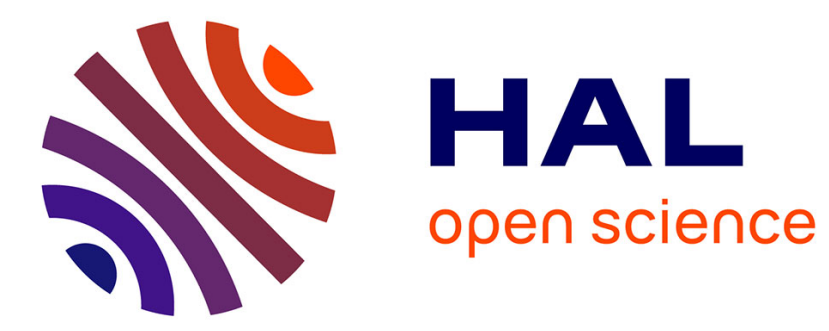

\title{
Specular reflection of matter waves from a rough mirror
}

Véronique Savalli, David Stevens, Jérôme Estève, P. D. Featonby, Vincent Josse, Nathalie Westbrook, Christoph I Westbrook, Alain Aspect

\section{To cite this version:}

Véronique Savalli, David Stevens, Jérôme Estève, P. D. Featonby, Vincent Josse, et al.. Specular reflection of matter waves from a rough mirror. Physical Review Letters, 2002, 88 (25), pp.250404. 10.1103/PhysRevLett.88.250404 . hal-00112250

\section{HAL Id: hal-00112250 \\ https://hal.science/hal-00112250}

Submitted on 12 Apr 2016

HAL is a multi-disciplinary open access archive for the deposit and dissemination of scientific research documents, whether they are published or not. The documents may come from teaching and research institutions in France or abroad, or from public or private research centers.
L'archive ouverte pluridisciplinaire HAL, est destinée au dépôt et à la diffusion de documents scientifiques de niveau recherche, publiés ou non, émanant des établissements d'enseignement et de recherche français ou étrangers, des laboratoires publics ou privés. 


\title{
Specular Reflection of Matter Waves from a Rough Mirror
}

\author{
V. Savalli, D. Stevens, J. Estève, P. D. Featonby, V. Josse, N. Westbrook, C. I. Westbrook, and A. Aspect \\ Laboratoire Charles Fabry de l'Institut d'Optique, UMR 8501 du CNRS, 91403 Orsay Cedex, France
}

(Received 18 February 2002; published 10 June 2002)

\begin{abstract}
We present a high resolution study of the specularity of the atomic reflection from an evanescent wave mirror using velocity selective Raman transitions. We observed a double structure in the velocity distribution after reflection: a peak consistent with specular reflection and a diffuse reflection pedestal whose contribution decreases rapidly with increasing detuning. The diffuse reflection is due to two distinct effects: spontaneous emission in the evanescent wave and roughness in the evanescent wave potential whose amplitude is smaller than the de Broglie wavelength of the reflected atoms.
\end{abstract}

DOI: $10.1103 /$ PhysRevLett.88.250404

Atomic mirrors are key components in the growing field of atom optics and have been intensively studied by several groups in recent years [1-9]. For interferometric and lithographic applications, it is important to ensure that the reflection is specular, since diffuse scattering amounts to a loss of spatial coherence and hence reduces fringe visibility or focusing sharpness. Thus much interest has been devoted to measuring and improving the roughness of atomic mirrors, using static or time dependent magnetic fields [1-4], evanescent waves [5], or other techniques [7].

Most of those experiments prepared a narrow velocity distribution, transverse to the direction of incidence, and measured the broadening of this distribution due to the reflection. The effect of the mirror was characterized by a single quantity, the rms increase in the width of the velocity distribution. This increase was sometimes given in terms of an effective rms angular deviation from a perfectly flat surface. However, the resolution allowed only a measurement of the broadening of the atomic velocity distribution. Here we present an experiment using velocity selective Raman transitions to prepare a very narrow initial velocity distribution [10] and measure the distribution after reflection. For the first time we are able to resolve not just the rms broadening but more complex structure in the final velocity distribution.

In analogy with the reflection of light from an optical mirror, we observe a double structure, with a narrow peak and a broad pedestal. The narrow peak corresponds to specularly reflected atoms, the broad pedestal to diffuse atomic reflection from two origins: spontaneous emission and mirror roughness. Assuming that the roughness of the mirror can be viewed as a random process with variance $\sigma^{2}$ and a correlation length much shorter than the mirror itself, one finds $[11,12]$ that the fraction $S$ of specularly reflected atoms is given by $\exp -w$, reminiscent of the Debye-Waller factor, with $w=16 \pi^{2} \sigma^{2} / \lambda_{\mathrm{dB}}^{2}$, where $\lambda_{\mathrm{dB}}$ is the de Broglie wavelength of the incident matter wave $(8 \mathrm{~nm})$. The presence of a significant specular peak implies that $\sigma \ll \lambda_{\mathrm{dB}}[13]$.

We have studied the ratio of these two components as a function of various parameters and show that the diffuse
PACS numbers: 03.75.Be, 32.80.Lg, 42.25.Fx, 42.50.Vk

component rapidly decreases when the evanescent wave detuning $\Delta_{\mathrm{EW}}$ increases. These observations allow us to examine different possible mechanisms for the diffuse reflection involving either mirror roughness or spontaneous emission.

The evanescent wave mirror was identical to the one described in Ref. [14]. We use a superpolished prism of TaFD30 glass [15] (refractive index $n_{1}=1.869$ ). The rms surface roughness given by the manufacturer is $0.07 \mathrm{~nm}$. The input and output faces are coated with a broadband antireflection coating. A Ti:S laser of wavelength $\lambda_{L}=$ $2 \pi / k_{L}=780 \mathrm{~nm}$ generates the evanescent wave with an incident angle $\theta_{1}=53^{\circ}$. The evanescent electric field thus has a decay constant of $\kappa=k_{L} \sqrt{n_{1}^{2} \sin ^{2} \theta_{1}-1}=1.11 k_{L}$ and a propagation vector of magnitude $k_{x}=k_{L} n_{1} \sin \theta_{1}=$ $1.49 k_{L}$. We have defined the $x$ axis to be along the evanescent wave propagation direction. The Ti:S beam is TM (p) polarized with waists $0.9 \mathrm{~mm}$ along the $x$ and $z$ axes at the prism surface.

Our ${ }^{85} \mathrm{Rb}$ magneto-optical trap (MOT) is described in Ref. [14]. Every $1.5 \mathrm{~s}$, we collect about $10^{8}$ atoms in the trap. By turning off the repumping beam just before (after) the trapping beams, we prepare the atoms in $5 S_{1 / 2}, F=2$ $\left(5 S_{1 / 2}, F=3\right)$. The MOT is situated $20 \mathrm{~mm}$ above the prism.

Velocity selective Raman transitions between $F=2$ and $F=3$ are induced by a pair of counterpropagating laser beams detuned by about $1 \mathrm{GHz}$ ( $\Delta$ in Fig. 1) [10]. Because of the Doppler effect, the resonance condition for a Raman transition depends on velocity and is given by $\delta=2 k\left(v+v_{\text {rec }}\right)$, where $\delta=\omega_{a}-\omega_{b}-\omega_{23}$ is the detuning of the Raman beams from the hyperfine transition, $v=\mathbf{v} \cdot \mathbf{k}_{a} / k_{a}$ is the projected atomic velocity, and $v_{\text {rec }}$ is the recoil velocity $(6 \mathrm{~mm} / \mathrm{s})$. By varying $\delta$, we select the velocity class that experiences a transition.

The two Raman beams are orthogonally linearly polarized and drive the magnetic field-independent transition between $\left|5 S_{1 / 2} F=2, m_{F}=0\right\rangle$ and $\left|5 S_{1 / 2} F=3, m_{F}=0\right\rangle$ denoted $|2\rangle$ and $|3\rangle$. We will use Dirac notation to refer to these two states, while the notation $F=2$ or 3 refers to the entire Zeeman manifold. A $750 \mathrm{mG}$ magnetic field 

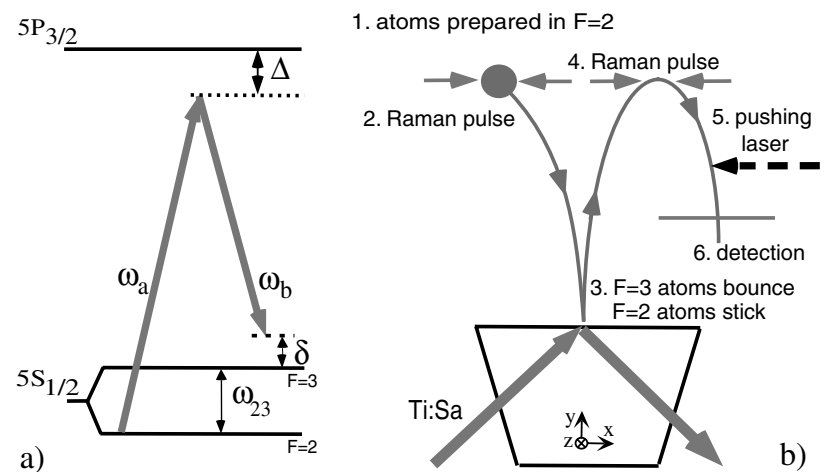

FIG. 1. (a) Level diagram for velocity selective Raman transitions. Frequencies $\omega_{a}$ and $\omega_{b}$ of the two counterpropagating laser beams are separated by ${ }^{85} \mathrm{Rb}$ hyperfine frequency $\omega_{23}$ plus Raman detuning $\delta$. (b) Sequence used in our experiment to select and analyze the velocity. The selection pulse is applied as the atoms fall toward the mirror. The analysis pulse comes at the top of their trajectory after bouncing.

oriented along the beam propagation direction (at $43^{\circ}$ to the $x$ axis in the $x z$ plane) lifts the degeneracy. Resonant transitions take place only between $|2\rangle$ and $|3\rangle$. To ensure that the atoms are in an eigenstate of the reflecting potential, we adiabatically rotate the magnetic field while they fall to be along the $z$ axis, the quantization axis defined by the evanescent wave electric field [14]. After reflection the magnetic field is turned back.

To generate the Raman beams (separated by $\omega_{23} / 2 \pi=$ $3.036 \mathrm{GHz}$ ), we modulate the injection current of a diode laser at $1.5 \mathrm{GHz}$ and inject the \pm 1 sidebands into two slave laser diodes. The carrier and \pm 2 sidebands are removed with a Fabry-Perot cavity. Each Raman beam passes through an acousto-optic modulator modulated by an arbitrary function generator to produce Blackman pulses [16]. Beating the two slave laser beams indicates a relative frequency spread less than $20 \mathrm{~Hz}$ (the resolution bandwidth of the spectrum analyzer) narrow enough not to limit the Raman transition width.

To test our setup, we first make a velocity selection and immediately analyze it with a second Raman pulse. At $t=$ 0 , we prepare the atoms in $F=3$. At $t=8 \mathrm{~ms}$ we apply a Raman "selection" $\pi$ pulse with a detuning $\delta_{\mathrm{S}}$ to transfer atoms to $|2\rangle$. Then comes a pushing beam resonant with the $5 S_{1 / 2} F=3 \rightarrow 5 P_{3 / 2} F^{\prime}=4$ transition which removes all the atoms remaining in $F=3$. At $t=22 \mathrm{~ms}$ we apply a second Raman "analysis" $\pi$ pulse with a detuning $\delta_{\mathrm{A}}$ to transfer atoms back to $|3\rangle$. Atoms in $F=3$ are detected via the fluorescence induced by a retroreflected probe laser resonant with the $5 S_{1 / 2} F=3 \rightarrow 5 P_{3 / 2} F^{\prime}=4$ transition and collected in a $0.1 \mathrm{sr}$ solid angle on a photomultiplier tube. No repumper is present in order to avoid detection of $F=2$.

We repeat the sequence changing $\delta_{\mathrm{A}}$ to acquire the transverse velocity distribution of incident atoms [Fig. 2(a)]. The rms width of the distribution is $7.3 \mathrm{kHz}$. This width is consistent with what is expected for a
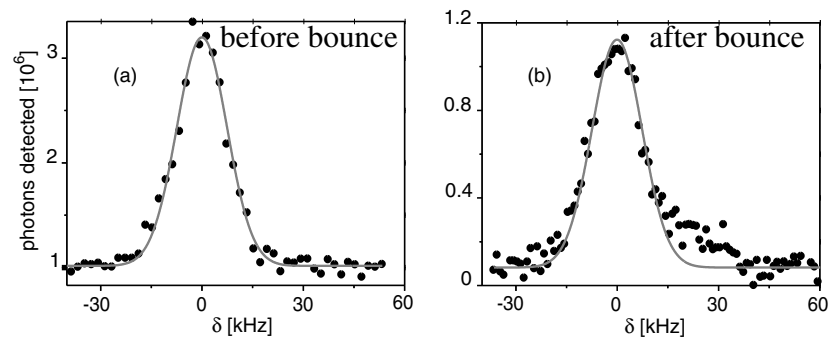

FIG. 2. Transverse atomic velocity distribution before (a) and after reflection (b) with $\Delta_{\mathrm{EW}}=2.4 \mathrm{GHz}$. The solid line of (a) is a Gaussian fit to the data (rms width $0.47 v_{\text {rec }}$ ). In (b), we have plotted the same Gaussian as in (a), normalized to the height of the data in (b) in order to emphasize the presence of a pedestal.

$150 \mu \mathrm{s}$ Blackman pulse. The curve demonstrates a velocity selection rms width for a single pulse of $0.33 v_{\text {rec }}$ along the propagation direction of the Raman beams. This is about 20 times narrower than the velocity width in the MOT. Because the analysis sequence has the same resolution as the selection sequence, our velocity resolution is $\sqrt{2}$ times larger, that is, $0.47 v_{\text {rec }}$. This resolution is 3 times better than that used in Ref. [5].

To observe the effect of the reflection on the transverse velocity, we proceed in a manner analogous to that described above (Fig. 1). At $t=0$ we prepare the atoms in $F=2$. The Raman selection pulse transfers a narrow velocity class to $|3\rangle$ at $t=8 \mathrm{~ms}$. The atoms then fall onto the mirror. The frequency of the evanescent wave is blue detuned for $F=3$ and red detuned for $F=2$. Atoms in $F=2$ are not reflected from the mirror. After reflection $(t=120 \mathrm{~ms})$, the analysis pulse transfers some atoms back into $|2\rangle$. Next, the pushing beam removes atoms remaining in $F=3$ and finally we detect atoms in $|2\rangle$ with probe laser and repumper. Compared to the test sequence, the roles of $F=2$ and $F=3$ are reversed in order to take advantage of the state selective nature of the reflection, as well as the fact that atoms can scatter more probe photons in the presence of the repumper.

Atoms not selected by the first Raman selection pulse can contribute to a background if they are pumped into $|3\rangle$ (by the evanescent wave, for example) during their trajectory. We measure this background using the same sequence described above and $\delta_{\mathrm{S}}$ tuned far from resonance. We alternate between normal and background measurements and subtract the background on each shot. The analysis detuning $\delta_{\mathrm{A}}$ is scanned randomly over the desired values, and we acquire and average about three measurements for each $\delta_{\mathrm{A}}$ to acquire a spectrum such as shown in Fig. 2(b). The peak value in Fig. 2(b) corresponds to about $10^{4}$ atoms detected per bounce. Typically the background was about $3 \times 10^{3}$ atoms. Despite this subtraction, we observe a nonzero background in Figs. 2 and 3. This background appears to be due to atoms reflected from the mirror that are pumped into $F=2$ after reflection.

With this system we acquired data such as that in Fig. 2(b) showing the velocity distribution (the number of 


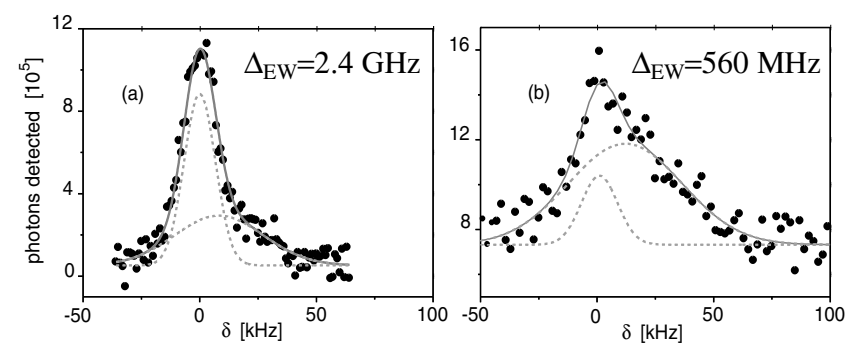

FIG. 3. Atomic velocity distribution for two $\Delta_{\mathrm{EW}}$ values. The solid lines show a fit using two Gaussian curves as described in the text. Both the individual Gaussians as well as their sum are shown. Each atom results in about 100 detected photons.

atoms detected in $F=2$ after the Raman analysis pulse) after the bounce. There is a narrow peak whose width appears identical to the initial one and a broad pedestal whose center is shifted by $7.9 \mathrm{kHz}$, an amount corresponding to a $0.5 \hbar k_{\mathrm{L}}$ momentum transfer with respect to the narrow one along the observation direction. This transfer is in the same direction as the evanescent wave propagation vector and remains so when the evanescent wave (Ti:S) laser direction is reversed (i.e., it also reverses).

To understand the origin of the pedestal, we acquired several reflected velocity distributions under differing conditions; two examples are shown in Fig. 3. Each distribution is fitted by a sum of two Gaussians plus a flat background. We first examined the parameters of the pedestal as a function of $\Delta_{\mathrm{EW}}$. We observed little variation of the width and the shift relative to the narrow peak. To simplify the study of the relative contribution of the two components, we fixed the width of the narrow peak at the measured width of the resolution function. We also fixed the width of the pedestal and the shift at the average values of our preliminary fits: the pedestal width was fixed to be that of the convolution of our resolution function and a Gaussian of $18 \mathrm{kHz}$ rms and the shift to be $7.9 \mathrm{kHz}$. Using this analysis we can measure the fraction $S$ of atoms detected in the narrow peak as a function of $\Delta_{\mathrm{EW}}$. (See Fig. 4.) The data are well fit by $S=\exp \left(-\alpha / \Delta_{\mathrm{EW}}\right)$ with $\alpha=1.1 \mathrm{GHz}$.

The above detuning dependence immediately suggests spontaneous emission within the evanescent wave which reduces the number of specularly reflected atoms by a factor of $\exp \left(-N_{\mathrm{SE}}\right)$. A simple estimate of $N_{\mathrm{SE}}$, the average number of spontaneous emissions, is given by $N_{\mathrm{SE}}=2 \pi /\left(\lambda_{\mathrm{dB}} \kappa\right) \times \Gamma / \Delta_{\mathrm{EW}}$, where $\Gamma / 2 \pi=5.9 \mathrm{MHz}$ is the natural linewidth of the atomic transition [9,17]. A better estimate includes the modification of the potential due to the van der Waals interaction [18], the modification of the spontaneous emission rate close to the surface [19], and an average of these effects over the mirror surface. We find, in our range of detunings, that $N_{\mathrm{SE}}$ still varies as $\Delta_{\mathrm{EW}}^{-1}$ to a good approximation but with a probability about 1.5 times higher than the simple estimate. To calculate $S$ one must also take into account the fact that at

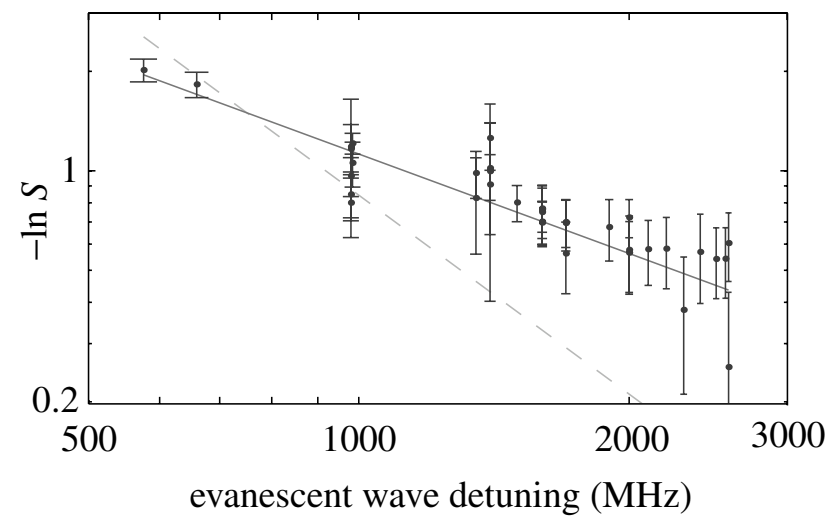

FIG. 4. Variation of $S$ the fraction of atoms in the narrow peak as a function of $\Delta_{\mathrm{EW}}$. The solid line (slope -1 ) shows $S=\exp -\alpha / \Delta_{\mathrm{EW}}$; the dashed line (slope -2 ) shows $S=\exp -\beta / \Delta_{\mathrm{EW}}^{2}$ with fitted $\alpha$ and $\beta$.

large detunings the branching ratio for falling back into $|3\rangle$, the only one which we detect, is $2 / 3$. This factor cancels the increase in $N_{\mathrm{SE}}$ due to the effects of the dielectric surface. One predicts therefore $S=\exp \left(-\alpha_{\mathrm{SE}} / \Delta_{\mathrm{EW}}\right)$ with $\alpha_{\mathrm{SE}}=0.55 \mathrm{GHz}$. There appears to be too little spontaneous emission (by a factor of 2) to entirely explain our results.

In addition, atoms undergoing spontaneous emission in the evanescent wave receive an average momentum transfer of $\hbar k_{x}$ along $x$, that is, a shift of the broad pedestal of $\hbar k_{x} \times \cos \left(43^{\circ}\right)=1.1 \hbar k_{\mathrm{L}}$ along the Raman beam direction instead of the $0.5 \hbar k_{\mathrm{L}}$ that we observe. [The distribution of spontaneously emitted photons, even in the presence of the dielectric surface is unchanged under $(x, y) \rightarrow(-x,-y)[19]$ and so only the absorption process contributes to the shift.] This observation confirms the above conclusion that spontaneous emission in the evanescent wave is only partly responsible for our observations. This is in contrast to the study of Ref. [9] which used a very small value of $\kappa$ to get a large number of spontaneous emissions in the evanescent wave.

Another mechanism which causes diffuse reflection is discussed in Ref. [12]. It involves scattered Ti:S light which interferes with the evanescent wave. The interference produces a rough potential which diffusely scatters the atoms. This mechanism does not involve spontaneous emission. The scattered light could come either from the surface roughness, inhomogeneities in the bulk of the prism, or some other object such as a prism edge. Using the results in Ref. [12] one can show that the propagating modes of the scattered light would cause $S$ to vary as $\exp \left(-\alpha_{\mathrm{R}} / \Delta_{\mathrm{EW}}\right)$, where $\alpha_{\mathrm{R}}$ depends on the amount of scattered light. The contribution to the pedestal due to this effect should exhibit no shift relative to the specular peak. Since spontaneous emission is responsible for about one-half of the pedestal, we expect a total pedestal shifted by about one-half of the shift due to spontaneous emission alone in good agreement with our observations. Since both 
effects have the same $\Delta_{\mathrm{EW}}$ dependence this explanation also accounts for the lack of detuning dependence in the observed shift.

Another possible explanation for the pedestal is spontaneous emission induced by the stray light above the prism while the atoms fall towards the mirror. Indeed, in experiments in which we left the evanescent wave laser on for $40 \mathrm{~ms}$ while the atoms fell towards the mirror, we observed an optical pumping of the atoms from their initial hyperfine level $(F=3)$ to the other hyperfine level $(F=2)$ (about $10 \%$ of the atoms were lost in this way at $\Delta_{\mathrm{EW}}=940 \mathrm{MHz}$ ). This mechanism predicts that $S$ should vary as $\exp -\beta / \Delta_{\mathrm{EW}}^{2}$, where $\beta$ is a constant which depends on the mean light intensity experienced by the atoms. As shown in Fig. 4 the data are not consistent with this dependence.

Thus we believe that we have identified the source of the diffuse reflection in our experiment as the sum of the effects of scattering of atoms from a potential induced by the random interference pattern of the evanescent wave and stray light, and spontaneous emission in the evanescent wave. According to this interpretation we have $1.1 \mathrm{GHz}=\alpha_{\mathrm{R}}+\alpha_{\mathrm{SE}}$, and we can work out the effective mirror roughness associated with $\alpha_{\mathrm{R}}$. We find $\sigma \approx 0.3 \mathrm{~nm}$, a value much larger than the prism's measured surface roughness $(0.07 \mathrm{~nm})$. Since the effective mirror roughness due to light scattering by the prism surface is of the order of the surface roughness itself [12], we presume that most of the stray light is from other sources such as those mentioned above.

We turn now to an analysis of the narrow peak. Since the area under the broad peak can be reduced by increasing the detuning $\Delta_{\mathrm{EW}}$, the essential question is "How faithfully is the initial velocity distribution reproduced in the narrow peak?" To answer this question, we compare the width of the narrow peak to that of the resolution function (atomic experimental velocity distribution before the bounce) for 36 runs acquired at different values of $\Delta_{\mathrm{EW}}$. We now fit the experimental curves by a sum of two Gaussians with all parameters adjustable except for the width and center of the broad peak. Averaging over 36 measurements, we find $\sigma_{\text {meas }}^{2}-\sigma_{\text {res }}^{2}=-\left(0.13 v_{\text {rec }}\right)^{2} \pm\left(0.08 v_{\text {rec }}\right)^{2}$, where $\sigma_{\text {res }}$ and $\sigma_{\text {meas }}$ are the rms widths of the two curves after the bounce, and the uncertainty is the standard deviation of the weighted mean of our 36 measurements. A negative sign in the result is not necessarily unphysical because it could be due, for example, to a slightly concave reflecting surface which collimates the atoms. We do not consider this deviation from zero to be statistically significant, however. We conclude that the observed reflection is consistent with a specular reflection to within about $0.1 v_{\text {rec }}$. Our limit is a factor of 10 better than our previous best result [5].

To compare our results with Refs. [1,3], we calculate the rms angular deviation of an effective reflecting surface from perfectly plane: $\sigma_{\theta}=\frac{1}{2} \frac{v_{\text {rms }}}{v_{\text {in }}}$, where $v_{\text {in }}$ is the incident atomic velocity on the mirror and $v_{\mathrm{rms}}$ is the rms transverse velocity added by the mirror. Using the upper limit $v_{\text {rms }}<0.1 v_{\text {rec }}$, we find that the effective mirror surface is flat to within uncertainty of $0.5 \mathrm{mrad}$.

We conclude that at sufficiently large detunings, it is possible to produce a highly specular mirror for atomic de Broglie waves. By analogy with photon optics, the double structure we observe suggests that we are in the regime where the roughness of the atomic mirror is small compared to the wavelength of the reflected matter wave. In that regime, the specular peak corresponds to a "perfectly" coherent reflection, and it should be possible to test this property in an atom interferometer. Interferometric experimental studies are in progress.

We acknowledge the assistance of C. Aussibal and thank C. Henkel and J. Thywissen for useful discussions. This work was supported by the European Union under Grants No. IST-1999-11055 and No. HPRN-CT-2000-00125 and by DGA Grant No. 99.34.050.

[1] C. V. Saba et al., Phys. Rev. Lett. 82, 468 (1999).

[2] L. Cognet et al., Europhys. Lett. 47, 538 (1999).

[3] D. Lau et al., Eur. Phys. J. D 5, 193 (1999).

[4] A. Arnold, C. MacCormick, and M. Boshier, Phys. Rev. A 65, 031601 (2002).

[5] A. Landragin et al., Opt. Lett. 21, 1591 (1996).

[6] K. Bongs et al., Phys. Rev. Lett. 83, 3577 (1999).

[7] I. Bloch et al., Phys. Rev. Lett. 87, 030401 (2001).

[8] D. Kadio, O. Houde, and L. Pruvost, Europhys. Lett. 54, 417 (2001).

[9] D. Voigt et al., Phys. Rev. A 61, 063412 (2000).

[10] M. Kasevich et al., Phys. Rev. Lett. 66, 2297 (1991).

[11] N. Garcia and M. Nieto-Vesperinas, Phys. Rev. Lett. 71, 3645 (1993).

[12] C. Henkel et al., Phys. Rev. A 55, 1160 (1997).

[13] Calculations relating the effective mirror roughness to substrate roughness are given in Refs. [11,12].

[14] L. Cognet et al., Phys. Rev. Lett. 81, 5044 (1998).

[15] From General Optics, Moorpark, CA.

[16] M. Kasevich and S. Chu, Phys. Rev. Lett. 69, 1741 (1992).

[17] M. Kasevich, D. Weiss, and S. Chu, Opt. Lett. 15, 607 (1990).

[18] A. Landragin et al., Phys. Rev. Lett. 77, 1464 (1996).

[19] J. Y. Courtois, J. M. Courty, and J. Mertz, Phys. Rev. A 53, 1862 (1996). 